\title{
Advantages of Beam Deceleration for Low kV EDS Analysis.
}

\author{
D. Edwards ${ }^{1}$, N. Erdman ${ }^{1}$, D. Guarrera ${ }^{1}$, M. Shibata ${ }^{1}$, V. Robertson ${ }^{1}$, F. Timischl ${ }^{2}$, Y. Nemoto $^{2}$ \\ 1. JEOL, USA Inc., 11 Dearborn Rd, Peabody, MA 01960 \\ 2. JEOL Technics Ltd, Akishima, Japan
}

Low voltage imaging and microanalysis have been gaining prominence in the last few years due to their distinct advantages for analysis of beam sensitive and charging materials. Recent advances in the SEM electron optics have allowed the SEM to maintain a small probe size at both imaging (small beam current) and analysis (large probe current) conditions at low accelerating voltages [1-3]. In particular in JEOL SEM microscopes one of the methods we have been employing to achieve this goal has been the use of beam deceleration (referred to as GB mode). Beam deceleration not only helps with charge balance but also effectively reduces lens aberrations thus improving overall image resolution. Gentle Beam works by retarding the primary beam voltage using a negatively charged stage bias to a lower landing energy. The landing voltage $\left(E_{\text {landing }}=E_{\text {gun }}-E_{\text {bias }}\right)$ can be varied with a combination of electron source voltage and specimen bias to achieve the necessary charge balance as well as high resolution performance at ultra-low voltages. The typical values for specimen bias are $0-2 \mathrm{kV}$, though better performance can be obtained with bias values up to $5 \mathrm{kV}$. Beam deceleration also serves as a form of aberration correction; the aberration coefficients (both spherical and chromatic) are reduced when the ratio $E_{\text {landing }} / E_{\text {gun }}$ is reduced for a fixed $E_{\text {gun }}$, meaning larger specimen bias enhances image resolution at ultra-low kVs. The use of Gentle Beam function thus preserves all the advantages of high $\mathrm{kV}$ imaging (gun brightness, small probe size) with added advantages of reduced charging, reduced specimen contamination and improved surface detail.

In this paper the benefits of beam deceleration at the specimen, as it applies to EDS will be demonstrated on the next generation Field Emission and Tungsten filament SEMs. Our extensive experience with imaging using Gentle Beam suggested that it would also be beneficial for elemental analysis of beam sensitive specimens that can't survive extensive counting times due to potential heat damage or specimens that require low voltage microanalysis because of charging. Figure 1 illustrates the improved elemental mapping that can be obtained by using a specimen bias. We have analyzed a sample of slag at $3 \mathrm{kV}$ and $90 \mathrm{nA}$ showing EDS mapping without application of GB (a,c,e) and with a $-5 \mathrm{kV}$ bias $(\mathrm{b}, \mathrm{d}, \mathrm{f})$. The maps demonstrate both enhancements in spatial resolution as well as signal to noise ratio when employing specimen bias.

We will further demonstrate application of this technique for composite samples, graphene, dopants in battery materials and nanoparticles on ITO. Our data will show that utilizing this technique the user could minimize acquisition time during EDS mapping while still maintaining good signal to noise ratio and high spatial resolution, making analysis of non-conductive and/or beam sensitive specimens more accessible.

References:

[1] [2] D.C. Bell and N. Erdman, in Low Voltage Electron Microscopy: Principles and Applications (2012): 1-30.

[2] N. Rowlands et al. Microscopy and Microanalysis 15.S2 (2009): 548-549.

[3] D. Edwards et al. Microscopy and Microanalysis 20.S3 (2014): 646-647. 

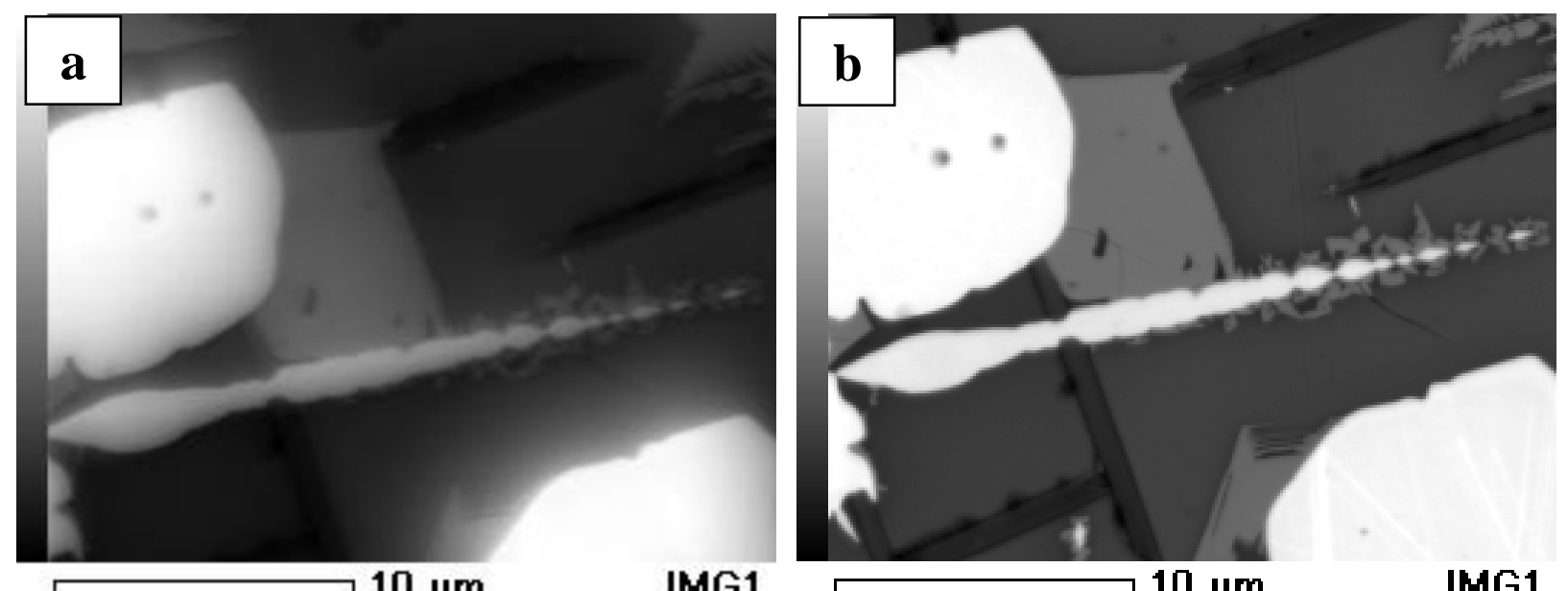

$10 \mu \mathrm{m}$

IMG1

$10 \mu \mathrm{m}$

IMG1
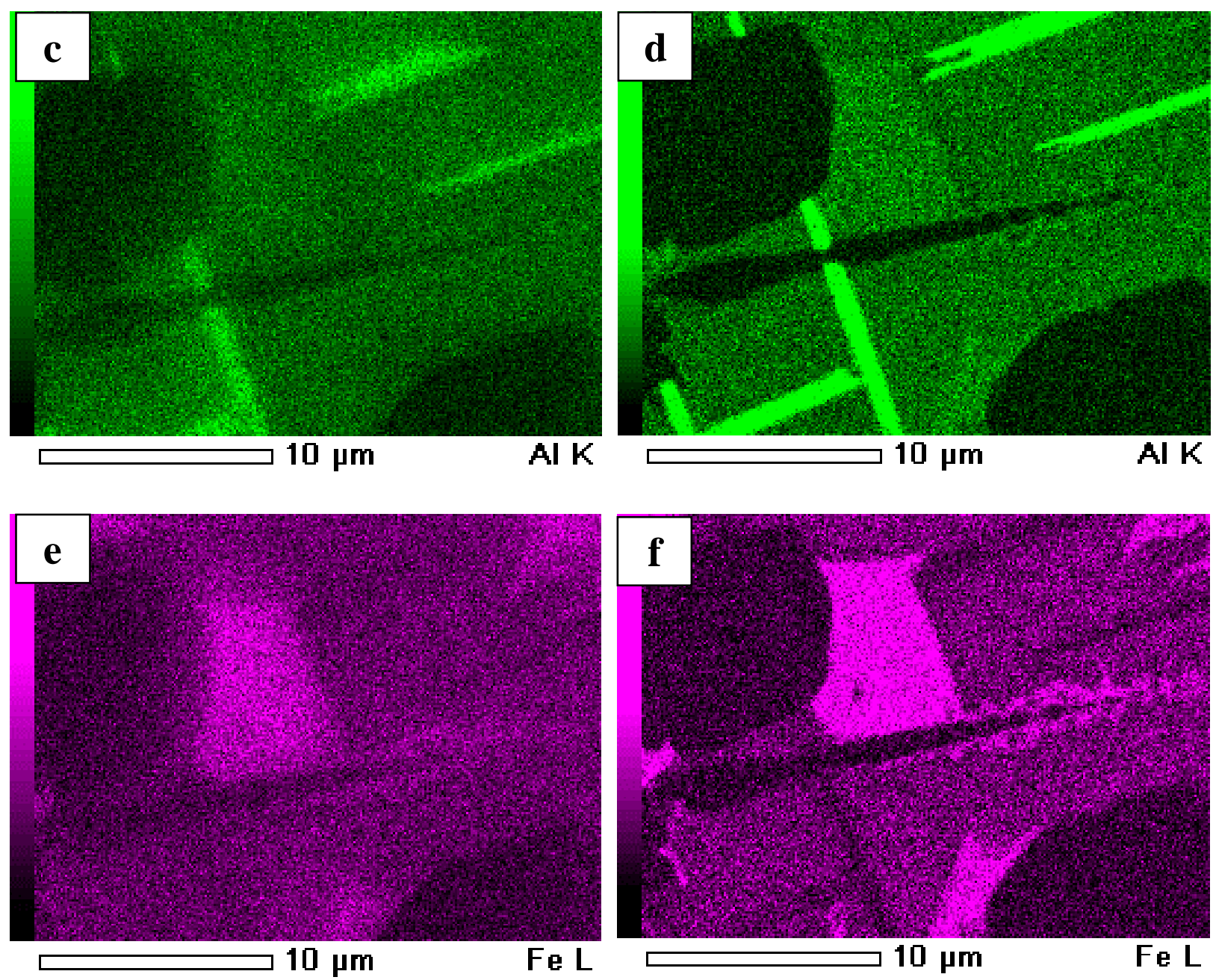

Figure 1. EDS map of Slag at 3kV and 90nA: (a,c,e) No Gentle Beam; (b,d,f) -5kV Gentle Beam. 